\title{
Does parathyroid hormone control bone quality?
}

\author{
Dolores Shoback ${ }^{1}$
}

Received: 8 December 2016 / Accepted: 26 December 2016 / Published online: 2 January 2017

(C) Springer Science+Business Media New York 2016

The article in this issue of Endocrine by Cipriani and colleagues from Columbia University in New York contrasts the effects of restoring toward normal the parathyroid hormone (PTH) levels in two cohorts of patients: one with primary hyperparathyroidism and the other with hypoparathyroidism [1]. Patients in these two groups approach the euparathyroid state from two vastly different places as regards bone mass and microarchitecture and levels of formation/resorption and cellular activity. Over the last two decades, this team of investigators led by Drs. Bilezikian, Silverberg and Rubin have tenaciously gone after the question of how PTH mediates changes in skeletal mass and structure and more elusive properties of the mineral and matrix components of bone that comprise its "quality" using primary hyperparathyroidism and hypoparathyroidism as model systems. Their efforts first focused on defining the clinical, biochemical, and densitometric hallmarks of primary hyperparathyroidism-a condition mainly seen today in a mild and asymptomatic form, sometimes presenting as the normocalcemic variant. Observations from their 15-year cohort study of 116 patients [2] shaped our current Guideline for assessing and managing patients with this disorder [3].

Their investigations next focused on the opposing condition-chronic hyposecretion of PTH. Properties of bone in this condition were largely unexplored until their work began. They assessed the effects of hypoparathyroidism on

Dolores Shoback

Dolores.Shoback@ucsf.edu

1 Endocrine Research Unit, San Francisco Department of Veterans Affairs Medical Center, University of California, San Francisco, CA 94121, USA skeletal mass and microarchitecture and bone quality by both classical (histomorphometry) as well as newer noninvasive skeletal phenotyping techniques (high-resolution microcomputed tomography, finite element analysis, trabecular bone score (TBS), and others) [4]. An understanding of the steady-state or baseline skeletal phenotype in hypoparathyroidism laid the foundation for Rubin and colleagues to examine the effects of replacing intact PTH on bone and other parameters in clinical trials. Their work provides an in-depth view of how bone adapts to chronic PTH deprivation and then how it responds to daily treatment with the missing hormone [5]. Their findings, combined with those from other groups [6, 7], helped support the US Food and Drug Administration's approval of recombinant human ( $\mathrm{rh}$ ) PTH (1-84) in 2015 for the treatment of patients with chronic hypoparathyroidism not controlled by conventional therapy (http://www.shirecontent.com/PI/PDFs/Natpara USA_ENG.pdf). This treatment is also expected to be available in Europe in 2017.

What insights does the current study of PTH's effects on bone offer? Cipriani et al. [1] contrast the chronic effects of two vastly different steady-state levels of PTH on bone at baseline. Intact PTH levels averaged $4 \mathrm{pg} / \mathrm{ml}$ in hypoparathyroid subjects vs. $115 \mathrm{pg} / \mathrm{ml}$ in those with primary hyperparathyroidism. Two parameters [dual energy xray absorptiometry (DXA) for bone mass and TBS for bone quality] were assessed over a 24-month period on the path to attaining more normal levels of PTH action in both groups of patients. The 52 hypoparathyroid subjects received daily rhPTH (1-84) injections, while 26 patients with primary hyperparathyroidism underwent curative surgery. With those interventions, both groups of patients were anticipated to equilibrate their bone parameters to what was now more normal levels of PTH action. 
Interesting and divergent skeletal responses became apparent in the two groups. In patients with hypoparathyroidism, bone mineral density (BMD) in the spine rose, reflecting an anabolic response to daily rhPTH (1-84). BMD at sites in the hip was unchanged over 24 months, while BMD of the distal radius, a site enriched with cortical bone, declined significantly. In contrast, return to normal circadian secretory patterns and levels of PTH in patients with primary hyperparathyroidism (achieved by the remaining native glands) produced significant positive effects on BMD at the spine and hip, but no changes at the distal radius. Differential responses between hyper- and hypoparathyroidism underscore the hypothesis that dosing of rhPTH (1-84) in hypoparathyroidism is likely to be in the catabolic range. This is because a key goal of treatment with hormone replacement in that condition is to achieve better control of hypocalcemia (with fewer supplements). This is likely to involve net mobilization of calcium, hence a catabolic dosing of PTH (1-84). In contrast, normal parathyroid glands constantly reset PTH secretion to maintain normocalcemia with ongoing readjustments in the renal production of 1,25-dihydroxyvitamin $\mathrm{D}$ and calcium reabsorption in response to fluctuating levels of PTH.

Divergent responses were also seen in TBS across the two patient groups. This noninvasive, DXA-based technique assesses spinal microarchitecture and is thought to reflect bone quality. TBS was greater at baseline in hypoparathyroid vs. hyperparathyroid subjects (1.44 vs. 1.28). Mean TBS was in the range of low fracture risk at baseline and improved significantly over time with rhPTH (1-84) treatment of hypoparathyroid subjects. This turned out not to be the case after curing the problem and restoring normal endogenous PTH secretion in patients with hyperparathyroidism. Their TBS scores were lower at baseline and did not significantly change after surgery.

Why were there differences in this measure of bone quality between hypoparathyroid and hyperparathyroid subjects being restored to more normal levels of PTH? The following explanations seem tenable. One is that vertebral bone in hypoparathyroidism, long dormant in a state of chronically low turnover, is demonstrating the anabolic responses to transient treatment (daily injections) with PTH. Such delivery of PTH allows for modeling and targeted remodeling to repair any accumulated microdamage and improve the integrity (quality) of trabecular bone. In contrast, during the recovery from hyperparathyroidism, perhaps closing the expanded remodeling space inherent to that disorder occurs in a more rapid time-frame than does the repair of degraded bone microarchitecture and quality. Hence, BMD improves promptly, but TBS does not. Perhaps the time required for new high-quality bone to be laid down and older bone to be replaced in the hyperparathyroid skeleton exceeds the 2 years of observation in this study.
Additionally, patients with primary hyperparathyroidism are often older estrogen-deficient females. At baseline, their trabecular microarchitecture has already deteriorated through a combination of aging and estrogen deficiency. That microarchitecture may never repair completely despite restoration of normal PTH levels. Additional studies will be needed to test that possibility.

What might this study tell us about the quality and strength of bone in hypoparathyroidism? Fracture rates are often used as clinical outcomes that reflect bone strength in a given clinical condition. Some studies suggest that vertebral fractures are increased in subjects with postsurgical [8] and idiopathic [9] hypoparathyroidism compared to controls. Population-based case control studies from Denmark, however, support the idea that vertebral fractures are not increased in postsurgical hypoparathyroid patients compared to controls [10], in keeping with what TBS data from this study would predict. As more hypoparathyroid patients receive PTH replacement in the years to come, it is anticipated that more precise estimates, by noninvasive techniques and by clinical fracture rates, will better inform the important issues of bone quality and strength in this disorder.

\section{Compliance with ethical standards}

Conflict of interest Dolores Shoback has received honoraria and consulting fees from Shire Pharmaceuticals and was a members of the European Society of Endocrinology guideline development committee and a member of the international guideline working group on hypoparathyroidism.

Ethical approval This article does not contain any studies with human participants or animals performed by any of the authors.

\section{References}

1. C. Cipriani, A. Abraham, B.C. Silva, N.E. Cusano, M.R. Rubin, D. J. McMahon, C. Zhang, D. Hans, S.J. Silverberg, J.P. Bilezikian. Skeletal changes after restoration of the euparathyroid state in patients with hypoparathyroidism and primary hyperparathyroidism. Endocrine (2016) doi:10.1007/s12020-016-1101-8 (pub online 18 October 2016)

2. M.R. Rubin, J.P. Bilezikian, D.J. McMahon, T. Jacobs, E. Shane, E. Siris, J. Udesky, S.J. Silverberg, The natural history of primary hyperparathyroidism with or without parathyroid surgery after 15 years. J. Clin. Endocrinol. Metab. 93, 3462-3470 (2008). doi:10. 1210/jc.2007-1215

3. J.P. Bilezikian, M.L. Brandi, R. Eastell, S.J. Silverberg, R. Udelsman, C. Marcocci, J.T. Potts Jr, Guidelines for the management of asymptomatic primary hyperparathyroidism: summary statement from the Fourth International Workshop. J. Clin. Endocrinol. Metabol 99, 3561-3569 (2014). doi:10.1210/jc.2014-1413

4. D.M. Shoback, J.P. Bilezikian, A.G. Costa, D. Dempster, H. Dralle, A.A. Khan, M. Peacock, M. Raffaelli, B.C. Silva, R.V. Thakker, T. Vokes, R. Bouillon, Presentation of hypoparathyroidism: etiologies and clinical features. J. Clin. Endocrinol. Metab. 101, 2300-2312 (2016). doi:10.1210/jc.2015-3909 
5. B.C. Silva, M.R. Rubin, N.E. Cusano, J.P. Bilezikian, Bone imaging in hypoparathyroidism. Osteoporos. Int. (2016). doi:10. 1007/s00198-016-3750-0

6. T. Sikjaer, L. Rejnmark, L. Rolighed L, L. Heickendorff, L. Mosekilde, the Hypoparathyroid Study Group, The effect of adding PTH (1-84) to conventional treatment of hypoparathyroidism: a randomized placebo-controlled study. J. Bone Miner. Res. 26, 2358-2370 (2011). doi:10.1002/jbmr.470

7. M. Mannstadt, B.L. Clarke, T. Vokes, M.L. Brandi, L. Ranganath, W.D. Fraser, P.L. Lakatos, L. Bajnok, R. Garceau, L. Mosekilde, H. Lagast, D. Shoback, J.P. Bilezikian, Efficacy and safety of recombinant human parathyroid hormone (1-84) in hypoparathyroidism (REPLACE): a double-blind, placebo-controlled, randomised phase 3 study. Lancet Diabetes Endocrinol. 1, 275-283 (2014). doi:10.1016/S2213-8587(13)70106-2
8. M.L. Mendonca, F.A. Pereira, M.H. Nogueira-Barbosa, L.M. Monsignore, S.R. Teixeira, P.C. Watanabe, L.M. Maciel, F.J. de Paula, Increased vertebral morphometric fracture in patients with postsurgical hypoparathyroidism despite normal bone mineral density. BMC Endocr. Disord. 13, 1 (2013). doi:10.1186/14726823-13-1

9. H. Chawla, S. Saha, D. Kandaswamy, R, Sharma, V. Sreenivas, R. Goswami, Vertebral fractures and bone mineral density in patients with idiopathic hypoparathyroidism on long term follow-up. J. Clin. Endocrinol. Metab. (2016) doi:10.1210/jc. 2016-3292

10. L. Underbjerg, T. Sikjaer, L. Mosekilde, L. Rejnmark, Postsurgical hypoparathyroidism-risk of fractures, psychiatric diseases, cancer, cataract, and infections. J. Clin. Endocrinol. Metab. 29, 2504-2510 (2014). doi:10.1002/jbmr.2273 